\title{
Los géneros Cajanus y Rhynchosia (Leguminosae, Papilionoideae, Phaseoleae, Cajaninae) en la península de Yucatán, México
}

\section{The genera Cajanus and Rhynchosia (Leguminosae, Papilionoideae, Phaseoleae, Cajaninae) in the Yucatán Peninsula, Mexico}

\author{
Angélica Martínez-Bernal ${ }^{1}$, Rodrigo Duno-de Stefano ${ }^{2 \otimes}$ y Lilia Lorena-Can $^{2}$ \\ ${ }^{l}$ Departamento de Biología, División de Ciencias Biológicas y de la Salud. Universidad Autónoma Metropolitana-Iztapalapa. Apartado postal 55-535, \\ 09340 México, D. F., México. \\ ${ }^{2}$ Herbario CICY, Centro de Investigación Científica de Yucatán, A. C. Calle 43. Núm. 130. Col. Chuburná de Hidalgo, Apartado postal 87, Cordemex \\ 97200 Mérida, Yucatán, México. \\ \roduno@cicy.mx
}

\begin{abstract}
Resumen. Se presenta información general de los géneros Cajanus DC. y Rhynchosia Lour. (subfamilia Papilionoideae, tribu Phaseoleae, subtribu Cajaninae) en la península de Yucatán. En esta región, Cajanus incluye 1 especie introducida y Rhynchosia incluye 6 nativas. Se presentan claves para géneros y especies, información ecológica, fenológica, nombres comunes y usos de cada especie.
\end{abstract}

Palabras clave: Fabaceae, flora, Mesoamérica, Provincia Biótica Península de Yucatán.

\begin{abstract}
An overview of the genera Cajanus DC. and Rhynchosia Lour. (subfamily Papilionoideae, tribe Phaseoleae, subtribe Cajaninae) in the Yucatan Peninsula is presented. In this region, the genus Cajanus includes 1 introduced species whereas Rhynchosia includes 6 native species. A key to the genus and the species known for the area is included as well as information about the ecology, phenology, common names and uses of each species.
\end{abstract}

Kew words: Fabaceae, flora, Mesoamerica, Yucatán Peninsula Biotic Province.

\section{Introducción}

Cajanus DC. y Rhynchosia Lour. son los únicos géneros de la subtribu Cajaninae, subfamilia Papilionoideae, tribu Phaseoleae (Lewis et al., 2005) presentes en la península de Yucatán. La subtribu incluye 10 géneros y cerca de 495 especies y su distribución es fundamentalmente paleotropical (Lewis et al., 2005). Hasta la fecha, se cuenta con varios listados florísticos; parciales o completos, para la porción mexicana de la península de Yucatán (e. g. Standley, 1930; Sousa y Cabrera, 1983; Sosa et al., 1985; Durán et al., 2000; Gutiérrez-Báez, 2000; Arellano-Rodríguez et al., 2003). En estas obras, el uso de los nombres no presenta mayor complicación con la excepción de $R$. pyramidalis (Lam.) Urb., especie que menciona Arellano-Rodríguez et al. (2003), la cual no crece en la región (véase sección de Nombres y especies excluidos al final del presente artículo). Duno-de Stefano et al. (2006) aclaran la presencia en la región del género Eriosema (también de la subtribu Cajaninae); Sosa et al. (1985) y Durán et al. (2000) registraron E. simplicifolium

Recibido: 18 agosto 2010; aceptado: 23 mayo 2011 basándose en el ejemplar de C. Chan y J. S. Flores 555 (CICY) que corresponde a Rhynchosia americana (Mill.) Metz. Hasta la fecha, no se ha registrado ninguna especie de Eriosema en la región de la península de Yucatán que corresponde a México.

El objetivo de este trabajo es presentar información general sobre Cajanus y Rhynchosia en la porción mexicana de la península de Yucatán, así como una clave para su identificación y comentarios generales de la morfología, fenología, ecología, nombres comunes y usos para cada una de las especies.

\section{Materiales y métodos}

Se revisó el material botánico de los herbarios más importantes de la región (CICY, CQRO, UADY, UCAM), así como el de otros herbarios nacionales (MEXU, UAMIZ, XAL) y extranjeros (HUH, LINN, MO, NY y US).

Los límites geográficos de la Provincia Biótica Península de Yucatán coinciden con los propuestos por Estrada-Loera (1991) y Tapia et al. (2007); en otras palabras, incluyen los estados mexicanos de Yucatán, Campeche y Quintana Roo, los distritos de Belize, Coro- 
Clave para los géneros de la subfamilia Papilionoideae, tribu Phaseoleae, subtribu Cajaninae en la península de Yucatán

1. Arbustos; hojas siempre 3-folioladas, folíolos laterales oblicuamente elípticos, marcadamente discoloros; flores con los lóbulos del cáliz desiguales, los lóbulos del cáliz más cortos que el tubo del cáliz; legumbres de 2 a $13 \mathrm{~cm}$ de largo, con 2-9 semillas; plantas cultivadas y ocasionalmente escapadas Cajanus 1. Hierbas trepadoras, rastreras, sufrútices o bejucos; hojas generalmente 3-folioladas y unifolioladas, folíolos generalmente ovados, rómbico-ovados hasta deltoides, ocasionalmente suborbiculares a reniformes; flores con los lóbulos del cáliz desiguales, en general los lóbulos del cáliz más largos, pero también se presentan más cortos y rara vez del mismo largo que el tubo del mismo; legumbres en general menos de $4 \mathrm{~cm}$ de largo, con 2 semillas; plantas nativas, en muchas ocasiones como malezas en campos de cultivo

Rhynchosia

zal y Orange Walk en Belice y el departamento del Petén en Guatemala. Los apartados Material examinado incluyen algunos ejemplares seleccionados de estos distritos y del departamento, pero en los mapas sólo se representan los de colecciones mexicanas.

\section{Descripción}

El género Cajanus, nativo del Paleotrópico, presenta una especie ampliamente cultivada en el Neotrópico; ocasionalmente es una planta escapada, pero nunca naturalizada. En el caso de Rhynchosia, existe un tratamiento taxonómico para las especies del Nuevo Mundo que ha sido fundamental para el conocimiento de los taxa en la península de Yucatán (Grear, 1978); dicho tratamiento incluye 5 especies. Una sexta especie, $R$. americana (Mill.) Metz fue registrada por primera vez en el área por Gutiérrez-Báez et al. (2000). Rhynchosia pyramidalis (Lam.) Urb., mencionada en fechas recientes (Arellano-Rodríguez et al., 2003), es una especie de amplia distribución en el Neotrópico, crece en el departamento de El Cayo en Belice y en el departamento del Petén en Guatemala, lo que corresponde a la porción más meridional de la Provincia Biótica Península de Yucatán, pero no crece en la parte mexicana.

Cajanus DC., Cat. Pl. Horti Monsp. 85. 1813, nom. cons. Cajan Adans., Fam. Pl. 2: 326. 1763.

Cantharospermum Wight et Arn., Prodr. Fl. Ind. Orient 255-256. 1834.

Atylosia Wight et Arn., Prodr. Fl. Ind. Orient. 257-258. 1834.

Endomallus Gagnep., Notul. Syst. (Paris) 3:184-186. 1915.

Peekelia Harms, Notizbl. Bot. Gart. Berlin-Dahlem 7:26[370]. 1920.

Arbustos de hasta $4 \mathrm{~m}$ de alto, perennes o anuales. Tallos acostillados cuando jóvenes, teretes cuando maduros. Hojas alternas, pinnadas o a veces digitadamente 3-folioladas; folíolos ligeramente desiguales, discoloros, membranáceos, los laterales oblicuamente elípticos, base cuneada, ápice agudo, densamente pubescentes en ambas caras, con glándulas vesiculares, especialmente en el envés; estípulas triangular-lanceoladas, caducas, estipelas presentes o ausentes. Inflorescencias racemosas, axilares o terminales, pedunculadas o casi sésiles; brácteas deciduas; bractéolas ausentes. Flores zigomorfas, 6-12 por raquis; brácteas pequeñas, caducas; cáliz campanulado, 5-lobado, lóbulos agudos o acuminados, el superior más largo, lóbulos más cortos que el tubo del cáliz; corola amarilla o el estandarte rojizo, estandarte ovado-orbicular, unguiculado, auriculado, hasta 3 cm de largo; alas oblicuamente obovadas; pétalos de la quilla auriculados, oblicuamente-redondeados, obtusos; estambres 10, diadelfos, el vexilar libre; ovario sésil, densamente pubescente y punteado-glandular; estilo delicado, glabro, curvado distalmente; estigma capitado. Legumbre linear-oblonga, 2-8(-13) $\mathrm{cm}$ de largo, 2 -valvada con constricciones oblicuas entre las semillas, amarillo-verdosa con manchas pardas, densamente indumentada, puberulenta a glabra, a veces con pelos glandulares; semillas 2-9, reniformes a suborbiculares, el hilo oblongo, estrofioladas.

Cajanus es un género con 34 especies (Lewis et al., 2005), nativo del Paleotrópico, pero una especie ha sido ampliamente introducida en todo el Neotrópico y sur de los Estados Unidos de América como planta comestible y ocasionalmente se puede encontrar escapada.

Cajanus cajan (L.) Huth, Helios 11:133. 1893. - Cytisus cajan L., Sp. Pl. 2:739. 1753. Tipo: India, herb. Linnaeus (LINN 912:7).

Cajanus cajan (L.) Millsp., Publ. Field Columbian Mus., Bot. Ser. 2:53. 1900. nom. illeg. superfl.-Cytisus cajan L., Sp. Pl. 2:739. 1753. Tipo: China: Yunnan: Huangjiaping, Dali, 1700 m, Handel-Mazzetti 6425 (lectotipo: BM, designado por Stearn 1972; isolectotipo: LINN).

Arbustos de 1-4 m de alto, perennes o anuales; hojas 3-folioladas, discoloras; folíolos laterales oblicuamente elípticos, 3.6-12.0 cm de largo, 1.3-4.5 $\mathrm{cm}$ de ancho, el terminal ovado-elíptico, elíptico a angostamente elíptico, $4.5-13.0 \mathrm{~cm}$ de largo, $1.4-5.5 \mathrm{~cm}$ de ancho, ápice agudo a angostamente agudo, base atenuada; cara abaxial velu- 
tina, con glándulas amarillas, cara adaxial velutina, con pocas glándulas amarillas; flores con el tubo del cáliz (3) 4-5 (-6) mm de largo, lóbulos más cortos que el tubo, desiguales; corola amarilla, con los pétalos de 14-20 $\mathrm{mm}$ de largo, el estandarte un poco más largo; legumbre linear-oblonga, 2-8 (-13) cm de largo, 0.4-1 (-1-7) cm de ancho, recta o falcada, comprimida, valvas generalmente pubescentes y con rayas moradas, 2-9 semillas, blancas, pardas, purpureas o casi negras, 4-9 $\mathrm{mm}$ de largo, 3-8 $\mathrm{mm}$ de ancho, estrofioladas.

Material examinado. México. Campeche: ejido Narciso Mendoza, km 33 al S de Xpujil, 18 13' 40" N, 89²7' 12" O, 240 m snm, 12 octubre 1997 (fr.), S. Ramírez 195 (CICY, MEXU, MO). Yucatán: ejido de Mucel de Pixoy, $20^{\circ} 43^{\prime} 30^{\prime \prime}$ N, $88^{\circ} 21^{\prime}$ 55" O, 6 diciembre 1981, C. Chan 1069 (CICY). Belice. Belize District: between Belize river Bridge and Belize Saw Mill on Northern Highway, 20 junio 1973 (fr.), J. D. Dwyer 11286 (NY).

Distribución y ecología. Ampliamente distribuida en la región como planta de cultivo. En México se cultiva en gran parte del país; crece fundamentalmente en vegetación secundaria y selva baja caducifolia perturbada. Para su permanencia, requiere ser introducida de manera continua. Comentarios taxonómicos. Especie cultivada por sus semillas comestibles. Se ha recolectado en flor de octubre a noviembre; en fruto, de octubre a diciembre. Localmente se conoce con los nombres de chícharo, chícharo de árbol, chícharo grande, lenteja, kán xrenteja y tsi tsiba (los 2 últimos en maya). Otros nombres muy frecuentes utilizados en los trópicos americanos son cinchoncho, guandúo y gandul.

Rhynchosia Lour., Fl. Cochinch. 425, 460. 1790, nom. cons.

Dolicholus Medik., Vorles. Churpfälz. Phys.-Ökon. Ges. 2:354. 1787, nom. rejic.

Arcyphyllum Elliott, J. Acad. Nat. Sci. Philadelphia 1:371. 1818.

Baukea Vatke, Linnaea 43:104. 1881.

Sufrútices, hierbas rastreras o trepadoras o bejucos. Tallos teretes a acostillados, puberulentos, vilosos, híspidos, pubescentes a glabros. Hojas 1-3 folioladas, subsésiles a largamente pecioladas; estípulas persistentes o tempranamente caedizas; estipelas pequeñas, persistentes hasta tempranamente caedizas o ausentes; folíolos 3-palmados, cara abaxial puberulenta, densamente pubescente, vilosa, velutina, híspida a glabra, ocasionalmente con glándulas vesiculares, cara adaxial puberulenta, pubescente, vilosa a glabra. Inflorescencias racemosas, axilares, simples o escasamente ramificadas, 1-muchas flores; brácteas pequeñas, persistentes o caedizas; bractéolas ausentes; cáliz campanulado, 5-dentado, los lóbulos más largos, iguales o más cortos que el tubo del cáliz, los 2 lóbulos superiores más o menos unidos, generalmente pubescentes y a veces glandulosos; corola amarilla, a veces con líneas pardas, púrpuras o rojas, estandarte obovado a orbicular, emarginado a apiculado, con 2 aurículas inflexas en la base, con una uña, externamente pubescente; alas estrechamente oblongas, con 1 aurícula, con 1 uña atenuada; quilla falcada, curvada en el ápice, con 1 uña; estambres 10, diadelfos; ovario sésil a subsésil, viloso; óvulos (1)2; estilo incurvado, filiforme, glabro; estigma pequeño. Legumbre con 2-semillas, comprimida o no, a veces falcada, variadamente constricta entre las semillas, puberulenta, pubescente, vilosa, hirsuta a glabrescente, ocasionalmente con vesículas glandulares, dehiscente; semillas subreniformes a suborbiculares, grises, pardas, negras, o rojas y negras, moteadas, lustrosas.

Rhynchosia es un género ampliamente distribuido en regiones cálidas y tropicales de todo el mundo, más abundante en América tropical y África y pocas especies se encuentran en América templada; incluye 230 especies (Lewis et al., 2005), 20 en México y 6 en la porción mexicana de la península de Yucatán (Grear, 1978).

Las especies de Rhynchosia del Nuevo Mundo han sido ubicadas en 2 secciones: Copisma (E. Mey.) Endl. y Arcyphyllum (Ellis) Torr. et Gray, conformadas por 6 series que parecen ser grupos homogéneos de especies relacionadas (Grear, 1978). Sin embargo, Fortunato (2000) asignó 8 especies neotropicales a la sección Rhynchosia, pero ninguna de estas especies está presente en la porción mexicana de la península de Yucatán. La sección Copisma incluye especies con tallos volubles, hojas trifolioladas y cáliz persistente hasta la fructificación, usualmente más corto que la corola, con lóbulos cortos y subulados, con legumbres y semillas diversas. Esta sección se divide en 4 series y en la península de Yucatán hay 3 especies: Rhynchosia minima (L.) DC. y $R$. swartzii (Vail) Urban en la serie 3 y $R$. yucatanensis Grear en la serie 4. Mientras que la sección Arcyphyllum incluye especies con tallos postrados o erectos, hojas unifolioladas o trifolioladas y el cáliz subfoliáceo a foliáceo, generalmente igual o más largo que la corola, con los lóbulos largos y lanceolados, las legumbres pequeñas, ovado-elípticas y las semillas suborbiculares. En el área de estudio esta sección está representada por 3 especies: $R$. americana (Mill.) Metz y $R$. reticulata (Sw.) DC. en la serie 5 y $R$. longeracemosa Mart. et Gal. en la serie 6 . 
Clave para las especies de Rhynchosia

1. Hojas unifolioladas, folíolos suborbiculares a reniformes Rhynchosia americana

1. Hojas 3-folioladas, folíolos nunca suborbiculares a reniformes. .2

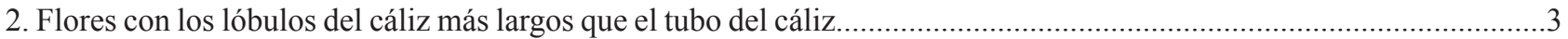

2. Flores con los lóbulos del cáliz generalmente más cortos o del mismo largo que el tubo del cáliz ...............................4

3. Tallos vilosos con pelos amarillos-dorados o rojizos, sin formar líneas evidentes en los ángulos del tallo; pecíolos 1.2-5.5 cm de largo; inflorescencias 3.5-14 cm de largo, más largas que las hojas ..... ..Rhynchosia longeracemosa 3. Tallos vilosos con pelos amarillos-dorados, formando líneas claras en los ángulos del tallo; pecíolos 1.0-3.5 cm de largo; inflorescencias 4.6-11.0 cm de largo, más cortas o iguales que el largo.de las hojas ..... Rhynchosia reticulata 4. Folíolos 2.0-7.0(-8.0) cm de largo, 1.7-5.5(-6.0) $\mathrm{cm}$ de ancho, rómbicos a deltoides, estípulas caedizas; inflorescencias 12.0-20.0 cm de largo; legumbre oblonga, 2.8-3.7 cm de largo, 0.6-0.8 $\mathrm{cm}$ de ancho Rhynchosia yucatanensis 4. Folíolos 0.9-6.5 cm de largo, 0.6-3.6 cm de ancho, ovados u ovado-rómbicos; estípulas persistentes; inflorescencias 4.0-15.0 cm de largo; legumbres oblongas de hasta $2.0 \mathrm{~cm}$ de largo o angostamente obovadas de $4.0 \mathrm{~cm}$ de largo ........5 5. Inflorescencias y cáliz con un solo tipo de pelo, simple y generalmente blanco; inflorescencias 4.0-15.0 cm de largo, generalmente más largas que las hojas; legumbre oblonga, ligeramente falcada, $1.5-2.0 \mathrm{~cm}$ de largo, $0.4-0.45 \mathrm{~cm}$ de ancho Rhynchosia minima

5. Inflorescencias y cáliz con 2 tipos de pelos, uno simple y generalmente blanco y otro más largo con la base ensanchada y amarillo; inflorescencias 6.0-7.0 cm de largo, más cortas que las hojas; legumbre angostamente obovada, 3.0-4.0 cm de largo, $0.7-0.8 \mathrm{~cm}$ de ancho .Rhynchosia swartzii

Rhynchosia americana (Mill.) Metz, Catholic Univ. Amer., Biol. Ser. 16: 126-127. 1934. (Fig. 7).

Lathyrus americanus Mill., Gard. Dict. (ed. 8) Lathyrus n. 19. 1768. como: "Americana".

Tipo: México. Veracruz: sin localidad exacta, W. Houstoun s.n., s.f. (holotipo: BM, no visto, NY-foto).

Hierbas rastreras o trepadoras; tallos teretes a ligeramente acostillados, pubescentes; pecíolos (1.0-)2.0-3.0 $(-5.0) \mathrm{cm}$ de largo; estípulas persistentes, 3.0-5.6 mm de largo; estipelas ausentes; hojas unifolioladas, único folíolo suborbicular a reniforme, $1.5-4.8 \mathrm{~cm}$ de largo, $1.5-4.1 \mathrm{~cm}$ de ancho, base cordada, ápice redondeado, cara abaxial puberulenta, más densa a lo largo del nervio principal y nervios secundarios, también vesículas glandulares, cara adaxial puberulenta; inflorescencias 2.0-3.0 cm de largo, más o menos iguales al largo de las hojas; cáliz 6.0-8.5 mm de largo, lóbulos más o menos iguales, más largos que el tubo del cáliz, viloso; corola amarilla frecuentemente con matices pardos, 6.0-10.0 $\mathrm{mm}$ de largo; legumbre ovada, 1.0-1.8 cm de largo, 0.5$0.7 \mathrm{~cm}$ de ancho, vilosa, base atenuada, ápice obtuso, rostro $10 \mathrm{~mm}$ de largo; semillas suborbiculares, comprimidas, pardas, negras o moteadas, 3.5-4.0 $\mathrm{mm}$ de largo, 3.0-3.8 $\mathrm{mm}$ de ancho.

Material examinado. México. Campeche: Puerto RicoAtasta, $18^{\circ} 37^{\prime} \mathrm{N}, 92^{\circ} 01^{\prime} \mathrm{O}, 0 \mathrm{~m}$ snm (fl., fr.), 28 mayo 1981, C. Chan y J. S. Flores 555 (CICY, MEXU, XAL); $5 \mathrm{~km}$ al E de Ciudad del Carmen, $18^{\circ} 38^{\prime} 30^{\prime \prime} \mathrm{N}, 91^{\circ} 45^{\prime}$ 55" O, $10 \mathrm{~m}$ snm (fl.), 27 julio 1996, C. Gutiérrez B. 5271 (MEXU, UCAM, UADY).
Distribución y ecología. Conocida en 2 localidades del estado de Campeche (Ciudad del Carmen y Puerto RicoAtasta) (Fig. 1). En México se ha registrado en Chiapas, Colima, Durango, Guerrero, Hidalgo, Querétaro, Oaxaca, San Luis Potosí, Sinaloa, Tabasco, Tamaulipas y Veracruz. También está registrada en los Estados Unidos de América (Texas) (Grear, 1978). Crece en manglar y selva mediana subcaducifolia perturbada.

Comentarios taxonómicos. Las 6 especies de Rhynchosia de la porción mexicana de la península de Yucatán reflejan claramente la existencia de 2 linajes morfológicos que corresponden a las secciones Copisma y Arcyphyllum. Rhynchosia americana es miembro de esta última sección junto con $R$. reticulata (serie 5) y $R$. longeracemosa (serie 6), pero se diferencia fácilmente de las anteriores por sus hojas unifolioladas suborbiculares o reniformes. Esta especie se conoce como planta forrajera (ganado bovino, caprino y equino). Su fenología en la península se conoce escasamente. Ha sido recolectada en flor y fruto en marzo, junio y julio.

Rhynchosia longeracemosa Mart. et Gal., Bull. Acad. Roy. Sci. Bruxelles 10(8):21. 1843. (Figs. 8a, b).

Tipo: México. Veracruz: cordillera, H. Galeotti 3322, junio-octubre 1840 (holotipo: K; isotipos: G, K000082222, K-000502994!, LE, P).

Hierbas trepadoras o bejucos; tallos acostillados, vilosos, con el tiempo se tornan puberulentos, con pelos amarillos-dorados o rojizos, sin formar líneas evidentes en los ángulos del tallo; pecíolos 1.2-5.5 cm de largo; estípulas tempranamente caedizas, $2.0-4.0 \mathrm{~mm}$ de largo; estipelas 

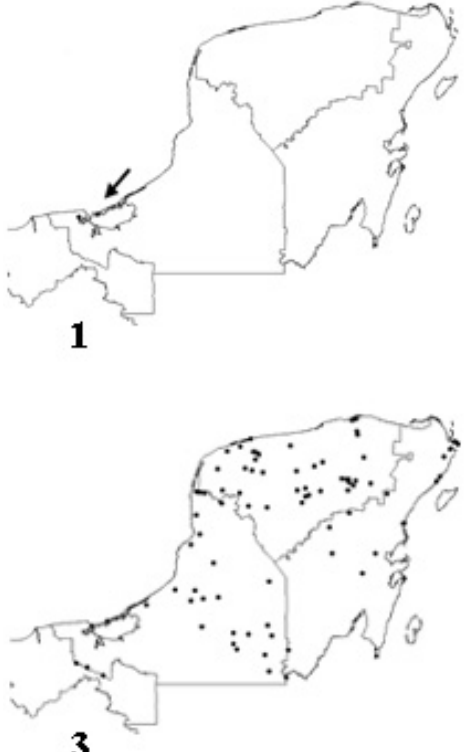

3

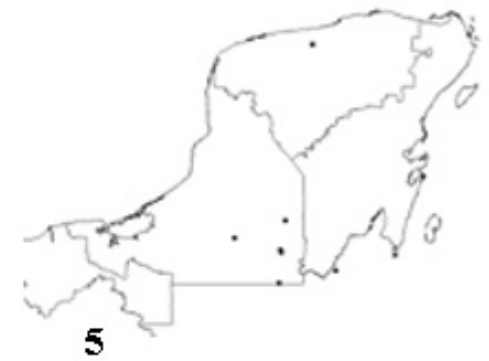

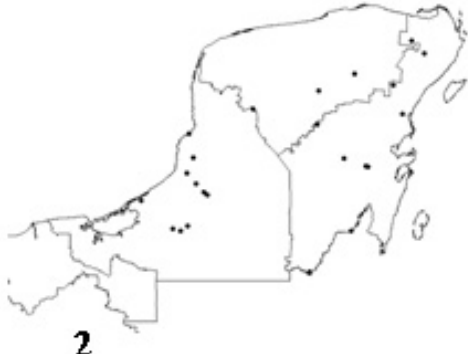

2

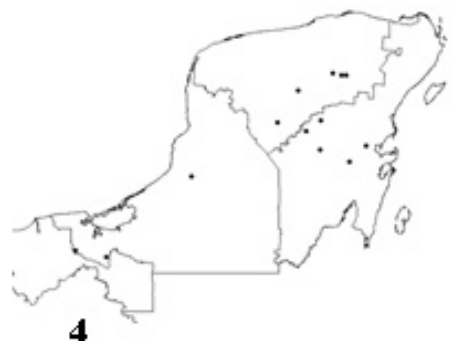

4

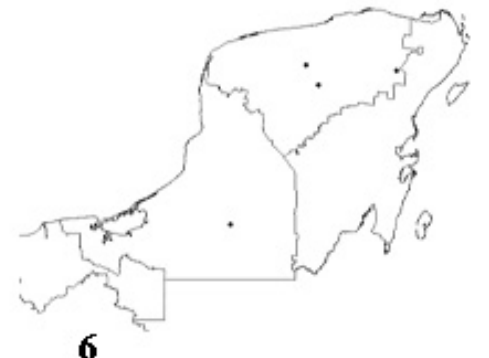

Figuras 1-6. Mapas de distribución de las especies del género Rhynchosia en la península de Yucatán. 1, Rhynchosia americana (Mill.) Metz.; 2, Rhynchosia longeracemosa Mart. et Gal.; 3, Rhynchosia minima (L.) DC.; 4, Rhynchosia reticulata (Sw.) DC. var. reticulata; 5, Rhynchosia swartzii (Vail) Urban; 6, Rhynchosia yucatanensis Grear. El mapa de Cajanus cajan (L.) Millsp. no se incluye, ya que se trata de una especie introducida, no naturalizada en la región.

2-5 mm de largo; hojas 3-folioladas, folíolos ovados a deltoides-ovados o rómbico-ovados, $2.0-8.0 \mathrm{~cm}$ de largo, 1.0-6.1 cm de ancho, base ampliamente redondeada, ápice agudo o acuminado, cara abaxial puberulenta a velutina en especial en las venas, cara adaxial puberulenta; inflorescencias (1.0-)3.5-14.0 cm de largo, en general más largas que las hojas; cáliz 6.5-10.0 mm de largo, lóbulos más o menos iguales, más largos que el tubo del cáliz, viloso; corola amarilla con bandas rojas, 6.8-11.0 mm de largo; legumbre ovada-oblonga, $1.9-2.5 \mathrm{~cm}$ de largo, 0.65-0.85 $\mathrm{cm}$ de ancho, densamente hirsuta a glabra, con pelos rojizos y vesículas glandulares, base atenuada, ápice agudo, rostro 55-72 mm de largo; semillas suborbiculares, comprimidas, pardas, negras o moteadas, 4.0-6.0 $\mathrm{mm}$ de largo, 3.0-4.0 $\mathrm{mm}$ de ancho.
Material examinado. México. Campeche: carretera Sabancuy-Díaz Ordaz, 8 febrero 1982 (fl), C. Chan 1177 (CICY, $\mathrm{XAL}$ ); $5 \mathrm{~km}$ al S de Yohaltún, 20 febrero 1981 (fr.), $C$. Chan 222 (CICY). Quintana Roo: camino a la laguna de Guerrero a Raudales, a $1 \mathrm{~km}$ de la laguna de Guerrero, 28 marzo 2006 (fl., fr.), R. Duno de Stefano et al. 2071, 2076 (CICY), cerca de la zona arqueológica Chacchoben, camino al pueblo de Lázaro Cárdenas a Limones, $4 \mathrm{~km}$ al NE del primero, 8 diciembre 2010 (fl.), R. Duno de Stefano \& G. Carnevali 2450, 2453 (CICY); zona arqueológica de Cobá, 20²9' 30" N, 89 43'55" O, 3 febrero 1980 (fr.), $O$. Téllez y E. Cabrera 1369 (CICY, MEXU). Yucatán: en los alrededores de la zona arqueológica de Sayil por la carretera Uxmal-Oxkutzkab, 20 10'07' N, 89 39'07” O, 25 diciembre 1985 (fl, fr.), E. Cabrera y H. de Cabrera 10344 


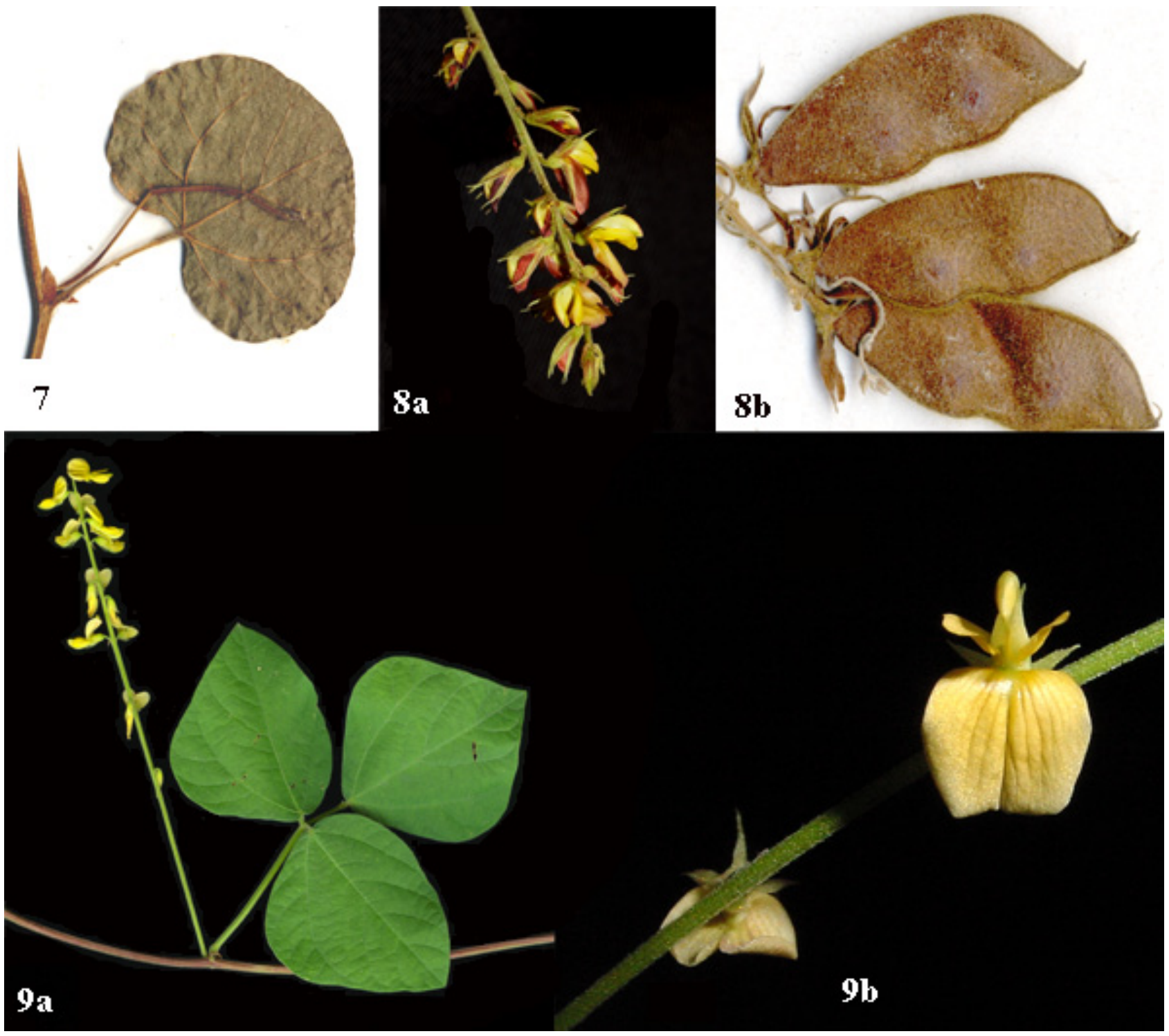

Figuras 7-9. Imágenes de algunas especies de Rhynchosia en la península de Yucatán. 7, Rhynchosia americana (Mill.) Metz (detalle de la hoja, C. Chan y J. S. Flores 555, CICY); 8a y 8b: Rhynchosia longeracemosa Mart. et Gal. 8a, detalle de la inflorescencia, $R$. Duno de Stefano \& G. Carnevali 2450, CICY, 8b, detalle del fruto, C. Chan 222, CICY;. 9a y 9b: Rhynchosia minima (L.) DC. 9a, detalle de hoja e inflorescencia, $9 \mathrm{~b}$, detalle de la flor.

(CICY, MEXU, CIQRO). Belice. Belize District: Old Northern Highway, mile marker $30^{\circ} 17^{\prime} 46^{\prime \prime} \mathrm{N}, 88^{\circ} 29^{\prime} \mathrm{O}$, 29 marzo 1990 (fl., fr.), R. Arvigo et al. 396 (NY).

Distribución y ecología. Ampliamente distribuida en toda la península de Yucatán (Fig. 2). En México se ha registrado en Chiapas, Hidalgo, Jalisco, Oaxaca, Puebla, Querétaro, San Luis Potosí, Tabasco, Tamaulipas y Veracruz. También crece en Guatemala y Belice hasta Costa Rica. Crece principalmente en vegetación secundaria y perturbada de selva baja caducifolia, selva mediana subcaducifolia y selva mediana subperennifolia.
Comentarios taxonómicos. Rhynchosia longeracemosa está incluida en la serie 6 de la sección Arcyphyllum (Grear, 1978). Esta especie es muy parecida a $R$. reticulata; $\sin$ embargo, esta última se ubica en la serie 5, junto con $R$. americana (Grear, 1978). Rhynchosia longeracemosa se ha mencionado como planta forrajera (ganado bovino, caprino y equino). Hasta la fecha ha sido recolectada en flor y fruto desde noviembre hasta marzo. Se conoce como bicho, y en maya ib cho.

Rhynchosia minima (L.) DC., Prodr. 2: 385. 1825. Dolichos minimus L., Sp. Pl. 726. 1752. (Figs. 9a, b). 
Tipo: Jamaica: St. Jago de la Vega, Sloane s.n., s.f. (lectotipo: BM, seleccionado por Verdcourt, citado por Grear, 1978).

Hierbas rastreras, trepadoras o bejucos; tallos ligeramente acostillados, puberulentos, con pelos blancos-plateados; pecíolos 0.8-3.2 cm de largo; estípulas persistentes, más tarde caedizas, $2.7-3.8 \mathrm{~mm}$ de largo, estipelas $0.5-1.0 \mathrm{~mm}$ de largo; hojas 3-folioladas, folíolos ovado-rómbicos a ovados, (1.1-) 2.2-4.6 (-5.0) cm de largo, 1.0-3.6 cm de ancho, base cuneada, obtusa a redondeada, ápice agudo, cara abaxial puberulenta y con vesículas glandulares, cara adaxial puberulenta a glabra; inflorescencias $4.0-15.0 \mathrm{~cm}$ de largo, igual o excediendo el largo de las hojas; cáliz 2.0-4.0 mm de largo, lóbulos desiguales, más o menos del mismo largo que el tubo del cáliz, puberulento, pelos simples blancos; corola amarilla, 5.0-7.0 $\mathrm{mm}$ de largo; legumbre oblonga, ligeramente falcada, $1.5-2.0 \mathrm{~cm}$ de largo, 0.4-0.45 $\mathrm{cm}$ de ancho, densamente puberulenta a glabrescente y con vesículas glandulares, base atenuada, ápice agudo, rostro 5-10 $\mathrm{mm}$ de largo; semillas ovadorenifomes, grises, pardas, negras o moteadas, 3.0-4.0 $\mathrm{mm}$ de largo, 2.0-3.0 $\mathrm{mm}$ de ancho.

Material examinado. México. Campeche: en el Remate, a $14 \mathrm{~km}$ al $\mathrm{O}$ de Tuncasche, sobre el camino a Punta Arenas, $20^{\circ} 32^{\prime} 01^{\prime \prime} \mathrm{N}, 90^{\circ} 22^{\prime}$ 04” O, 01 diciembre 1988 (fl., fr.), E. Cabrera y H. de Cabrera 15334 (CICY, MEXU). Quintana Roo: Ejido Caobas, Sabanas de Jaguactal, un desvió de $9.5 \mathrm{~km}$ por carretera de terracería al oeste de la carretera hacia Tres Garantías, unos $21 \mathrm{~km}$ al sur de la carretera principal Xpujil-Chetumal, $18^{\circ} 18^{\prime} 00^{\prime \prime} \mathrm{N}, 89^{\circ}$ 07’00” O, 30 abril 1999 (fl.), G. Carnevali 5520 (CICY). Yucatán: sin localidad exacta, s.f. (fl.), G. F. Gaumer 498 (US); sin localidad exacta, 1895 (fl.), G. F. Gaumer 768 (US); Chichankanab, s.f. (fr.), G. F. Gaumer 1366 (US); Chichankanab, s.f. (fl.), G. F. Gaumer 1945 (US); Calotmul, s.f., (fl.), G. F. Gaumer 1946 (US); Kancabdzonot, Febrero 1917 (fl., fr.), G. F. Gaumer 23576 (US); sin localidad exacta, 1917-1921 (fl.), G. F. Gaumer 24093 (US); $2 \mathrm{~km} \mathrm{~W}$ of Chemax, 20 39' $35^{\prime \prime} \mathrm{N}, 87^{\circ} 57^{\prime} 40^{\prime \prime} \mathrm{O}$, 8 marzo 1980 (fl., fr.), C. D. Johnson 1751-80 (CICY, MEXU). Guatemala. Petén: El Paso, 27 abril 1932 (fl.), C. L. Lundell 1569 (US); Parque Nacional Tikal, 12 julio 1969 (fl.), R. T. Ortiz 191 (US); ident, 25 agosto 1969 (fl., fr.), R. T. Ortiz 244 (US); 2 abril 1970 (fl., fr.), $R$. T. Ortiz 876 (US). Belice. Belize District: Western Highway, $17^{\circ} 28^{\prime} 40^{\prime \prime} \mathrm{N}, 88^{\circ} 14^{\prime} 48^{\prime \prime}$ O, 26 enero 1970 (fl.), D. L. Spellman 1495 (MO). Orange Walk: San Antonio, $18^{\circ}$ $07^{\prime} \mathrm{N}, 88^{\circ} 40^{\prime} \mathrm{O}, 25$ noviembre 1976 (fl.), P. W. Leino 7628 (MO).

Distribución y ecología. Ampliamente distribuida en toda la península de Yucatán (Fig. 3). En México se ha registrado en Baja California Sur, Chiapas, Chihuahua,
Coahuila, Colima, Durango, Guerrero, Guanajuato, Hidalgo, Jalisco, México, Michoacán, Morelos, Nayarit, Nuevo León, Oaxaca, Puebla, Querétaro, San Luis Potosí, Sinaloa, Sonora, Tabasco, Tamaulipas, Veracruz y Zacatecas. Es la especie de más amplia distribución desde Estados Unidos (Alabama, Florida, Georgia, Louisiana y Texas) hasta Argentina (Grear, 1978; Isley, 1990: Woods y Key, 2009). Crece virtualmente en todo tipo de vegetación, sobre todo en áreas perturbadas y es la especie más común en la península de Yucatán.

Comentarios taxonómicos. Rhynchosia minima presenta una gran variabilidad morfológica, sobre todo en el tamaño de la hoja y del indumento. Esta especie junto con $R$. swartzii y $R$. yucatanensis son miembros de Rhynchosia sección Copisma. En la clave que presentamos se resumen las diferencias más importantes de estas especies. Rhynchosia minima se cita como planta comestible y forrajera (ganado caprino, equino, vacuno y bovino). Ha sido recolectada en flor y fruto durante todo el año. Existe una larga lista de nombres comunes para esta planta, muchos simples variantes ortográficas: bu'ul bach, buul bech, icho, $i$ cho, ib ch'o, ib cho, bicho, ib-cho, lib ch'o', lib cho', mehenib-bech', $x$-ib cho y xixhilak (maya) y frijolillo, ibes e ibes del ratón (español).

Rhynchosia reticulata (Sw.) DC. var. reticulata, Prodr. 2: 385. 1825. Glycine reticulata Sw., Prodr. 105. 1788.

Tipo: Jamaica: sin localidad exacta, Swartz s.n., s.f. (lectotipo: S, seleccionado por Grear, 1978).

Hierbas trepadoras o bejucos; tallos acostillados, vilosos, con pelos amarillos-dorados, formando líneas o bandas en los ángulos del tallo; pecíolos 1.0-3.5, estípulas caedizas, $3.0-4.0 \mathrm{~mm}$ de largo; estipelas $1.0-1.5 \mathrm{~mm}$ de largo; hojas 3-folioladas, folíolos ovados, ovado-rómbicos u ovadooblongos, 2.5-7.5 cm de largo, 1.1-3.7 cm de ancho, base redondeada, obtusa, subcordada o cuneada, ápice agudo, acuminado, cara abaxial densamente vilosa a velutina, principalmente en las venas, cara adaxial puberulenta $\mathrm{o}$ vilosa; inflorescencias 4.6-11.0 cm de largo, más cortas o iguales al largo de los hojas; cáliz 5.5-7.0 mm de largo, lóbulos más o menos iguales, más largos que el tubo del cáliz, densamente viloso; corola amarilla, $5.0-7.5 \mathrm{~mm}$ de largo; legumbre oblonga-obovada, $1.7-2.5 \mathrm{~cm}$ de largo, 0.6-0.8 $\mathrm{mm}$ de ancho, puberulenta a glabrescente, especialmente en las suturas, con vesículas glandulares, base atenuada, ápice agudo, rostro $55 \mathrm{~mm}$ de largo; semillas suborbiculares, comprimidas, negras-pardas o moteadas, 4.0-5.0 mm de largo, 3.0-5.0 $\mathrm{mm}$ de ancho.

Material examinado. México. Campeche: San Joaquín rumbo a Jonuta. Palizada, $18^{\circ} 07^{\prime} 00^{\prime \prime} \mathrm{N}, 92^{\circ} 07^{\prime} 00^{\prime \prime} \mathrm{O}, 19$ febrero 1986 (fl.), C. Chan 6152 (CICY). Quintana Roo: laguna de Chichankanab, carretera Presumida-Sto. Tomás, $19^{\circ} 52^{\prime} 36^{\prime \prime} \mathrm{N}, 88^{\circ} 46^{\prime} 14^{\prime \prime}$ O, 25 marzo 1984 (fl.), J. J. 
Ortiz 463 (CICY-2). Yucatán: Temax, 21 09' 00” N, 89 01'12" O, 19 diciembre 1985 (fl.), E. Cabrera y H. de Cabrera 10002 (MEXU), Kancabdzonot, marzo 1917 (fl.), G. F. Gaumer 23605 (US); sin localidad exacta, 1917-1921 (fi.), G. F. Gaumer 24122 (US); frente a la ex hacienda San Miguel, 2043'02" N, 88¹7'20" O, 25 marzo 1983 (fl., fr.), E. Ucan 2318 (CICY).

Distribución y ecología. Ampliamente distribuida en toda la región (Fig.4). En México se ha registrado en Chiapas, Colima, Guerrero, Jalisco, Michoacán, Nayarit, Oaxaca, San Luis Potosí y Veracruz. Asimismo, en Nicaragua hasta Sudamérica y las Antillas (Grear, 1978). Una segunda variedad, $R$. reticulata var. kuntzei (Harms) Grear se distribuye en Centro América (El Salvador hasta Panamá) y Sur América (Colombia, Venezuela, Bolivia y Brasil) (Grear, 1978). Crece en selva baja caducifolia, selva mediana subperennifolia perturbada y vegetación secundaria.

Comentarios taxonómicos. Rhynchosia reticulata es miembro de la serie 5 de Rhynchosia sección Arcyphyllum, junto con $R$. americana (Grear, 1978). Sin embargo, el indumento y morfología del cáliz hacen que esta especie sea más afín morfológicamente a $R$. longeracemosa, ubicada en la serie 6 de la misma sección. Una segunda variedad, $R$. reticulata var. kuntzei (Harms) Grear, no registrada en México, se diferencia básicamente por sus estipulas persistentes, ovadas, foliáceas, con la base subcordada y hasta 8 $\mathrm{mm}$ de largo, mientras que la variedad típica tiene estipulas caedizas, lanceoladas, estrechamente ovadas con la base truncada y hasta $5 \mathrm{~mm}$ de largo (Grear, 1978). Rhynchosia. reticulata ha sido recolectada en flor entre febrero y abril, luego en septiembre y diciembre y en fruto, entre febrero-abril y luego en diciembre. Esta especie se menciona como medicinal para heridas e hinchazones y como forrajera para ganado bovino y caprino. Se conoce con los nombres comunes hulk'in, $x$-hulk'in y $x$-hulkin (maya).

Rhynchosia swartzii (Vail) Urban, Repert. Spec. Nov. Regni Veg., 15:320. 1918. Dolicholus swartzii Vail, Bull. Torrey Bot. Club 26(3):108-111. 1899.

Tipo: Estados Unidos de América. Florida. Key West, Blodgett s.n., s.f. (lectotipo: NY, seleccionado por Grear, 1978, isolectotipos: GH, NY!).

Hierbas trepadoras, sufrútices o bejucos; tallos teretes, híspidos, con tricomas blancos-plateados, con algunos pelos glandulosos; pecíolos 0.7-3.5 cm de largo; estípulas persistentes, 2-4 mm de largo; estipelas diminutas, no mayores de $1 \mathrm{~mm}$ de largo; hojas 3-folioladas; folíolos ovados u ovadorómbicos, 0.9-6.5 cm de largo, 0.6-2.8 cm de ancho; ápice agudo o ligeramente acuminado, base obtusa, redondeada o subtruncada, caras abaxial y adaxial híspidas; inflorescencias 6.0-7.0 cm de largo, generalmente no excediendo el largo de las hojas; cáliz 2.7-3.8 mm de largo, lóbulos más o menos iguales, más cortos que el tubo del cáliz, con 2 tipos de pelos, uno es simple y generalmente blanco y el otro con la base ensanchada es amarillo; corola amarilla, 5.0-8.0 mm de largo; legumbre angostamente obovada, 3.0-4.0 cm de largo, 0.7-0.8 cm de ancho, hirsuta, con algunas vesículas glandulares, base atenuada, ápice agudo, rostro de 10-35 mm de largo; semillas reniformes, comprimidas, rojas, 4.0$6.0 \mathrm{~mm}$ de largo, 3.0-3.5 $\mathrm{mm}$ de ancho.

Material examinado. México. Campeche: a $1 \mathrm{~km}$ al $\mathrm{N}$ de poblado Narciso Mendoza, $18^{\circ} 14^{\prime} 48^{\prime \prime} \mathrm{N}, 89^{\circ} 27^{\prime} 10^{\prime \prime} \mathrm{O}$, 169 m snm, 21 enero 1998 (fl., fr.), D. Álvarez 623 (CICY, MEXU); $18^{\circ} 41^{\prime} 30^{\prime \prime} \mathrm{N}, 89^{\circ} 24^{\prime} 13$ ' O, 270 m snm, 30 abril 1998 (fl.), E. M. Lira C. et al. 773 (MO); Tuxpeña, $18^{\circ} 27^{\prime}$ 00" N, 90 04' 48” O, 28 enero 1932 (fl., fr.), C. L. Lundell 1264 (GH, CICY-foto, US); $17^{\circ} 49^{\prime} 20^{\prime \prime} \mathrm{N}, 089^{\circ} 29^{\prime} 06^{\prime \prime}$ O, $200 \mathrm{~m}$ snm, 17 febrero 1998 (fl., fr.), E. Martínez S. et al. 30164 (MEXU, MO), $33 \mathrm{~km}$ al S de Xpujil; $18^{\circ} 17^{\prime} 20^{\prime \prime}$ N 89॰ 27' 55" O, 33 m snm, 12 octubre 1997 (fl., fr.), S. Ramírez A. 189 (MO). Quintana Roo: a $52 \mathrm{~km}$ al oeste de Felipe Carrillo Puerto o a $11 \mathrm{~km}$ de San Luis o a las afueras de Polyuc, 6 febrero 1980 (fl.), O. Téllez 1481 (CIQRO). Belice. Orange Walk District: $18^{\circ} 00^{\prime} \mathrm{N}, 88^{\circ} 42^{\prime} \mathrm{O}, 100 \mathrm{~m}$ snm, 19 marzo 1987 (fl.), G. Davidse y A. E. Brant 32799 (MO).

Distribución y ecología. Rhynchosia swartzii se ha recolectado sólo en el sur del estado de Campeche, Quintana Roo y una recolección dudosa en Yucatán (Temax). En México sólo crece en la península de Yucatán (Fig. 5). Se conoce de los Estados Unidos de América (Florida), Belice, Bahamas y Cuba. Crece en selva baja caducifolia perturbada, selva baja inundable, selva mediana subcaducifolia y selva alta perennifolia.

Comentarios taxonómicos. Rhynchosia swartzii puede confundirse con $R$. minima, ya que las hojas y flores son muy parecidas. Ambas especies se ubican en la serie 3 de la sección Copisma. Sin embargo, R. swartzii se puede distinguir porque sus inflorescencias, pedicelos y el cáliz están cubiertos con 2 tipos de pelos: uno simple de color blanco y otro más largo, con la base ensanchada y de color amari1lo. Sin duda un estudio micromorfológico de la superficie de hojas y flores revelaría claramente diferencias patentes en la morfología de los pelos que recubren estas estructuras. Rhynchosia swartzii se ha recolectado en flor entre enero y mayo, y luego en octubre; y en fruto, entre enero y mayo. Forrajera para ganado bovino y caprino. No se ha registrado ningún nombre común en el área de la península de Yucatán.

Rhynchosia yucatanensis Grear, Mem. New York Bot. Gard. 31(1):103-105, f. 27. 1978.

Tipo: México. Yucatán: sin localidad exacta, G. F. Gaumer 24332, 1917-1921 (holotipo: F; isotipo: US).

Hierbas trepadoras, sufrútices o bejucos; tallos teretes a acostillados, velutinos, con tricomas amarillo-dorados; 
pecíolos $1.3-7.5 \mathrm{~cm}$ de largo; estípulas tempranamente caedizas, 4-6 $\mathrm{mm}$ de largo; estipelas 2.5-5.0 $\mathrm{mm}$ de largo; hojas 3-folioladas; folíolos rómbicos a deltoides, 2.0-7.0($8.0) \mathrm{cm}$ de largo, 1.7-5.5(-6.0) $\mathrm{cm}$ de ancho, base obtusa a subtruncada, ápice agudo a acuminado, cara abaxial puberulenta a glabra, con vesículas glandulares, cara adaxial puberulenta a glabra; inflorescencias $12.0-20.0 \mathrm{~cm}$ de largo; cáliz de 3.0-4.0 $\mathrm{mm}$ de largo, lóbulos desiguales, iguales o más cortos que el tubo del cáliz; corola amarilla, 5.0-7.0 $\mathrm{mm}$ de largo; legumbre oblonga, 2.8-3.7 $\mathrm{cm}$ de largo, 0.6-0.8 cm de ancho, densamente puberulenta, con vesículas glandulares, base atenuada, ápice agudo, rostro 7-12 mm de largo; semillas reniformes, negras, pardas, o moteadas, 4.5-5.0 $\mathrm{mm}$ de largo, 3.0-3.5 $\mathrm{mm}$ de ancho.

Material examinado. México. Campeche: $8 \mathrm{~km}$ al E de Constitución, camino a Conhuas, $18^{\circ} 35^{\prime} 21^{\prime \prime} \mathrm{N}, 90^{\circ} 03^{\prime}$ 39” O, 128 m snm, 4 marzo 1998 (fr.), E. Martínez S. 30194 (MEXU, MO). Quintana Roo: a $27 \mathrm{~km}$ al norte de Cobá, 87 36'00" N, 20 41' 10" O, 12 marzo 1980 (fl. fr.), O. Téllez 1845 (CICY, MEXU, MO, CIQRO). Yucatán: sin localidad exacta, 1917-1921, G. G. Gaumer 24332 (F, no visto US).

Distribución y ecología. Rhynchosia yucatanensis se conoce de unas pocas localidades de Campeche, Quintana Roo, Yucatán, y también en Guatemala (Fig. 6). Crece en selva mediana subperennifolia perturbada. Sin embargo, se ha registrado en el estado de Chiapas (e.g. E. Cabrera y H. de Cabrera 7909, MO). El ejemplar chiapaneco presenta hojas y folíolos muy grandes. Se recomienda realizar otro tipo de estudios para saber si este material botánico pertenece a $R$. yucatanensis. Por lo anterior, esta especie aparentemente es endémica de la península de Yucatán.

Comentarios taxonómicos. Grear (1978) incluyó a esta especie en la serie 4 de la sección Copisma. El autor mencionó como especies relacionadas a $R$. nipenses Urban en la diagnosis y luego a $R$. rojasii Hassler en la discusión. La primera es una especie antillana (Cuba) con hojas bicolores muy distintivas y frutos y semillas muy pequeñas. La segunda es una especie sudamericana que crece en Paraguay, Brasil y Argentina con hojas muy grandes (e.g. folíolos 5-15 x 3-11 $\mathrm{cm}$, Grear, 1978). Sin embargo, por su distribución geográfica y morfología posiblemente Rhynchosia yucatanensis se relaciona más con $R$. minima, ya que esta última especie se asigna a la serie 3 de la misma sección Copisma. R. yucatanensis se diferencia de $R$. minima porque es una planta más robusta, con pecíolos, estípulas, estipelas, hojas, frutos y semillas más grandes. Su fenología se conoce escasamente y sólo ha sido colectada en fruto, en mayo. Esta especie también se registra como planta forrajera.

Nombres y especies excluidos

Rhynchosia pyramidalis (Lam.) Urb. Esta especie fue registrada como Rhynchosia. pyramidata (Lam.) Urb. por
Arellano-Rodríguez et al. (2003), evidentemente una mala trascripción del nombre correcto. También la mencionó Grear (1978) en la península de Yucatán (Maskall Pine Ridge, 19 febrero 1934, Gentle 1128 (F, GH, MICH, MO, NY). Sin embargo, esa localidad corresponde al distrito de Belice en ese mismo país. En México esta especie crece en Oaxaca, Tabasco y Veracruz. También se conoce de Guatemala, Honduras y en las Antillas.

\section{Agradecimientos}

A los curadores de los herbarios MEXU, UADY y UCAM, quienes permitieron estudiar el material disponible. La visita a MEXU de Rodrigo Duno-de Stefano fue financiada por el CICY (Centro de Investigación Científica de Yucatán, A. C.) en el marco del proyecto Sistemática de la familia Leguminosae en la Península de Yucatán (R-18). Los mapas de distribución se generaron con la ayuda del SIG de Biótica 4.3 (CONABIO). Al Dr. Fernando Chiang por sus valiosos comentarios.

\section{Literatura citada}

Arellano-Rodríguez, J. A., J. S. Flores-Guido, J. Tun-Garrido y M. M. Cruz-Bojórquez. 2003. Nomenclatura, forma de vida, uso, manejo y distribución de las especies vegetales de la Península de Yucatán. In Etnoflora Yucatanense 20. Universidad Autónoma de Yucatán. Mérida, Yucatán. 815 p.

Duno-de Stefano, R., L. Rico-Arce, A. Martínez-Bernal y C. Gutiérrez-Báez. 2006. Notes on the Flora of the Yucatan Peninsula V: New records and miscellaneous notes for the family Leguminosae. Boletín de la Sociedad Botánica de México 78:43-46.

Durán, R., G. Campos, J. C. Trejo, P. Simá, F. May Pat y M. Juan Qui. 2000. Listado Florístico de la Península de Yucatán, Centro de Investigación Científica de Yucatán, Mérida. 259 p.

Estrada-Loera, E. 1991. Phytogeographic relationships of the Yucatan Peninsula. Journal of Biogeography 18:687-697.

Fortunato, R. H. 2000. Systematic relationships in Rhynchosia (Cajaninae - Phaseoleae-Papilionoideae - Leguminosae) from the neotropics. In Advances in legume systematics. Part 9, P. S. Herendeen y A. Bruneau (eds.). Royal Botanic Gardens, Kew, Richmond, Surrey. p. 339-354.

Grear, J. W. 1978. A revision of the New World species of Rhynchosia (Leguminosae-Faboideae). Memoirs of the New York Botanical Garden 31:1-168.

Gutiérrez-Báez, C. 2000. Listado florístico actualizado del estado de Campeche, México. Universidad Autónoma de Campeche, Campeche. 95 p.

Isley, D. 1990. Vascular flora of the southeastern United States, vol. 3, part 2. Leguminosae (Fabaceae). The University of North Carolina Press, Chapel Hill. 258 p. 
Lewis, G., B. Schrire, B. Mackinder y M. Lock. 2005. Legumes of the world. The Royal Botanic Gardens, Kew, Richmond, Surrey. 577 p.

Standley, P. C. 1930. Flora of Yucatan. Field Museum of Natural History Publications. Botanical Series 3:157-492.

Sosa, V., J. Salvador-Flores, V. Rico-Gray, R. Lira y J. J. Ortiz. 1985. Lista florística y sinonimia maya. In Etnoflora Yucatanense 1: Instituto Nacional de Investigaciones sobre Recursos Bióticos, Xalapa, Veracruz. 225 p.

Sousa, S. M. y E. F. Cabrera. 1983. Listados florísticos de
México. II. Flora de Quintana Roo. Instituto de Biología, UNAM, México, D. F.

Tapia, J. L., G. Carnevali Fernández-Concha, R. Duno-de Stefano, I. M. Ramírez-Morillo, S. Hernández-Aguilar y L. Lorena Can-Itza. 2007. Notas sobre la Flora de la Península de Yucatán VII: Boehmeria cylindrica (Urticaceae), un nuevo registro para la región. Boletín de la Sociedad Botánica de México 81:141-143.

Woods, M. y J. Key. 2009. The genus Rhynchosia (Fabaceae) in Alabama. Phytologia 91:3-17. 